\title{
Paracoccidioidomicosis juvenil diseminada diagnosticada en una niña en área urbana
}

\author{
Angélica Ballesteros, Sandra Beltrán, Jaime Patiño, Cynthia Bernal, Rocío Orduz \\ Departamento de Pediatría, Clínica Universitaria Colombia, Bogotá, D.C., Colombia
}

\begin{abstract}
Introducción. La paracoccidioidomicosis es una de las infecciones micóticas sistémicas más prevalente en los países latinoamericanos. La tasa de incidencia ha ido en incremento y su detección es cada vez más común en viajeros o inmigrantes de las zonas endémicas. Se caracteriza por un cuadro de síntomas respiratorios, linfadenopatías y lesiones cutáneas que progresan paulatinamente, por lo que la enfermedad crónica no tratada puede llevar a la muerte.

Objetivo. Describir el enfoque clínico y diagnóstico de una enfermedad tropical exótica en una zona urbana. Caso clínico. Se describe un caso de paracoccidioidomicosis juvenil en área urbana con un cuadro clínico de síndrome febril de un mes de evolución, compromiso ganglionar generalizado, manifestaciones cutáneas y pérdida de peso; el examen físico evidenció episodio febril y hepatoesplenomegalia, cuyo diagnóstico fue difícil. Se inició manejo con itraconazol y trimetoprim-sulfametozaxol con posterior cese de los picos febriles y mejoría significativa de las lesiones en la piel.

Se hizo una revisión extensa de la literatura científica con los criterios de búsqueda de Pubmed adaptados para las diferentes bases de datos; la revisión se basó en los estudios hallados en Medline, LILACS, SciELO y Cochrane Library desde 1966 hasta la fecha. Los estudios fueron seleccionados por los autores con base en su relevancia y aporte científico a la discusión del caso en mención.

Conclusiones. Son pocos los casos de paracoccidioidomicosis en la población pediátrica a pesar de ser endémica en América Latina. Las crecientes corrientes de urbanización de las zonas endémicas hacen que este cuadro deba sospecharse.
\end{abstract}

Palabras clave: Paracoccidioides, paracoccidioidomicosis, eosinofilia, micosis.

doi: http://dx.doi.org/10.7705/biomedica.v34i1.1637

\section{Disseminated juvenile paracoccidioidomycosis diagnosed in a girl in an urban area}

Introduction: Paracoccidioidomycosis is one of the most prevalent systemic fungal infections in Latin American countries. The incidence rate has been increasing and its detection has gotten increasingly common in travelers or immigrants from endemic areas. It is characterized by respiratory symptoms, lymphadenopathies and skin lesions, which gradually progress and subsequently lead to death in some untreated chronic disease cases.

Objective: To describe the clinical approach and diagnosis of an exotic tropical pathology in an urban area. Case description: Case description and extended literature review.

We made a case report of urban paracoccidioidomycosis in a young patient, with a clinical syndrome of fever for a month, widespread nodal involvement, cutaneous manifestations and weight loss. During the physical examination, hepatosplenomegaly and a febrile episode were evidenced, whose diagnosis was difficult. Management started with itraconazole and trimethoprim sulfamethoxazole, with subsequent cessation of spiking fevers and significant improvement of the skin lesions.

We performed a comprehensive literature review, with search criteria performed in PubMed and adapted for different databases. The review was conducted based on the studies found in Medline, LILACS, SciELO and Cochrane Library, from 1966 up to this moment. Studies were selected by the authors based on their relevance and scientific contribution to the discussion of this case.

Conclusions:There are few cases of paracoccidiomycosis in the pediatric population despite the endemicity of this entity in Latin America. An increasing population influx from endemic areas makes suspecting of this disease a must.

Key words: Paracoccidioides, paracoccidiomycosis, eosinophilia, mycosis. doi: http://dx.doi.org/10.7705/biomedica.v34i1.1637

\footnotetext{
Contribución de los autores:

Angélica Ballesteros: desarrollo de la estructura del manuscrito y revisión de la literatura.

Sandra Beltrán: diagnóstico clínico del caso y revisión de la literatura.

Jaime Patiño: seguimiento del caso y revisión de la literatura.

Cynthia Bernal: revisión de la literatura.

Rocío Orduz: diagnóstico histopatológico.
} 
La paracoccidioidomicosis, anteriormente conocida como blastomicosis suramericana, granuloma paracoccidioidico o enfermedad de Lutz-SplendoreAlmeida, es una enfermedad granulomatosa causada por el hongo dimorfo Paracoccidioides brasiliensis $(1,2)$.

Se la considera una enfermedad endémica restringida a los países latinoamericanos, de poca prevalencia en los países de Centroamérica. La mayoría de los casos se ha reportado en Brasil (80 $\%$ ), con una tasa estimada de 1,45 casos por cada millón de habitantes, donde la región central y norte del país reportan tasas de prevalencia elevadas, seguido por Venezuela, Colombia, Ecuador y Argentina (3). La infección tradicionalmente afecta a la población que vive en las áreas rurales con predominio en agricultores, quienes se infectan regularmente con Paracoccioides spp.; sin embargo, en los últimos años se ha detectado una tendencia a su incremento en la población cercana al área urbana $(1,4)$.

El hombre es el huésped principal; sin embargo, la micosis se ha descrito en perros y armadillos, los cuales pueden estar infectados naturalmente. La vía de entrada principal de este microorganismo es la respiratoria, aunque muy pocos de las personas infectadas presentan síntomas clínicos; se sabe que para generar la enfermedad sistémica es necesario un compromiso de la respuesta inmunitaria del huésped (5). En la forma aguda, la infección tiene un pico de incidencia entre los 10 y 20 años de edad, aunque las manifestaciones clínicas se aprecian frecuentemente en adultos entre los 30 y 50 años de edad como reactivación de un foco latente. Los casos de paracoccidioidomicosis en edad pediátrica representan solamente de 5 a $10 \%$ de todos los casos y en ellos predomina la forma aguda y subaguda sin distinción de sexo, a diferencia de lo que ocurre en adultos en quienes la distribución es de 10 a 15 hombres por una mujer (3).

\section{Presentación del caso}

Se trata de un paciente de sexo femenino de 12 años de edad, natural de Bogotá, que consultó por haber presentado fiebre de $39{ }^{\circ} \mathrm{C}$ durante un mes, pérdida de $5 \mathrm{~kg}$ de peso, aproximadamente,

\section{Correspondencia:}

Angélica Ballesteros, Departamento de Pediatría, Fundación Universitaria Sanitas, Calle 22B N ${ }^{\circ}$ 66-46, Bogotá, D.C., Colombia.

angelik210@gmail.com

Recibido: 18/06/13; aceptado: 24/10/13

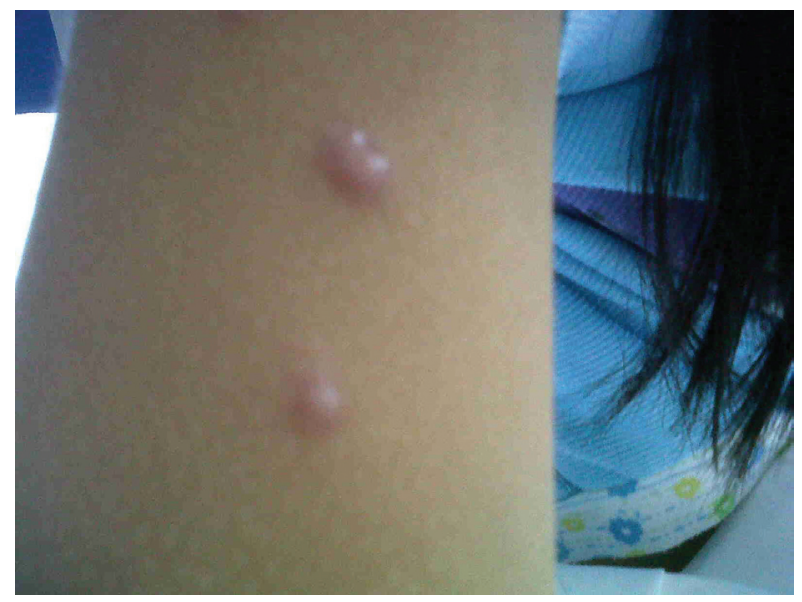

Figura 1. Lesiones en antebrazo (Servicio de Pediatría de la Clínica Universitaria Colombia)

adenopatías generalizadas de uno a dos centímetros de diámetro, indoloras, y múltiples lesiones pápulo-vesiculares indoloras, esféricas y con depresión central (figura 1).

No presentaba antecedentes médicos de importancia; residía en zona urbana, cerca de un humedal, el que negó frecuentar; también negó convivencia con animales y salidas de la ciudad o nexos de importancia.

Al ingreso presentaba fiebre y hepatoesplenomegalia. En el hemograma realizado el mes anterior se encontró leucocitosis y eosinofilia. Durante la hospitalización persistió la fiebre y en el día 15 de fiebre aparecieron lesiones en la piel de las extremidades y en el abdomen. La paciente presentó caquexia, adinamia, astenia e hiporexia.

Los exámenes de laboratorio para parasitosis, enfermedad autoinmune, tuberculosis e inmunoglubulinas $A$, G y $\mathrm{M}$ resultaron normales. La IgE estaba elevada y el perfil autoinmune normal. En la tomografía de cuello, tórax y abdomen se observaron adenopatías generalizadas. La baciloscopia de esputo y la PPD resultaron negativas; los anticuerpos para Leptospira spp., Toxocara catis y Toxocara canis fueron negativos, así como el VIH. El ecocardiograma fue normal, y la PCR para el gen $B C R / A B L$ dio negativo.

Ocho días después del ingreso, la paciente continuaba febril, y sin cambios en las lesiones de piel ni en el tamaño de las adenopatías. Se sospechó síndrome de hipereosinofilia y se solicitaron exámenes para establecer la subpoblación de linfocitos $\mathrm{T}$ y $\mathrm{B}$, la presencia de células asesinas naturales y de antígeno 
galactomanano. Se inició manejo con itraconazol y trimetoprim-sulfametoxazol. Dos días después cesaron los picos febriles y hubo mejoría significativa de las lesiones de piel.

En el aspirado de médula ósea se encontró eosinofilia y conteo bajo de blastos. La biopsia de las lesiones en piel se informó inflamación granulomatosa crónica y presencia de estructuras micóticas. El diagnóstico se realizó mediante la visualización de las estructuras fúngicas típicas en la biopsia del ganglio (figura 2) y se confirmó por técnicas moleculares con extracción de ADN del tejido del ganglio en parafina, usando como gen control el de la betaglobina, y una amplificación con una PCR panfúngica (común en los hongos), la cual fue positiva. Posteriormente se hizo secuenciación del hongo amplificado, se comparó con los bancos de datos y, finalmente, se identificó plenamente como $P$. brasiliensis (Corporación para Investigaciones Biológicas, Medellín).

Por otra parte, se inspeccionó el sitio de residencia de la paciente ubicado cerca al humedal de La Conejera en Bogotá, incluida la vivienda y las cercanías del humedal. No se realizó muestreo del hongo.

Pasado un año de seguimiento la paciente permanece asintomática con la dosis adecuada de itraconazol; los niveles séricos han permanecido en el rango subterapéutico, y la respuesta clínica ha sido satisfactoria. Después de 10 meses de tratamiento, las pruebas de serología fueron negativas para $P$. brasiliensis.

\section{Revisión de la literatura}

Se realizó una revisión amplia de la literatura científica relacionada con el caso clínico presentado en este estudio, la cual incluyó la búsqueda de estudios relevantes publicados en las siguientes bases de datos: Medline, LILACS, SciELO y revistas locales no indizadas. Se estructuró la siguiente estrategia de búsqueda, adaptada para cada una de las bases de datos: ("paracoccidioides" [MeSHTerms] OR "paracoccidioides" [AllFields]) AND ("child" [MeSHTerms] OR "child" [AllFields] OR "children" [AllFields]). No se restringió la búsqueda por año ni por idioma.
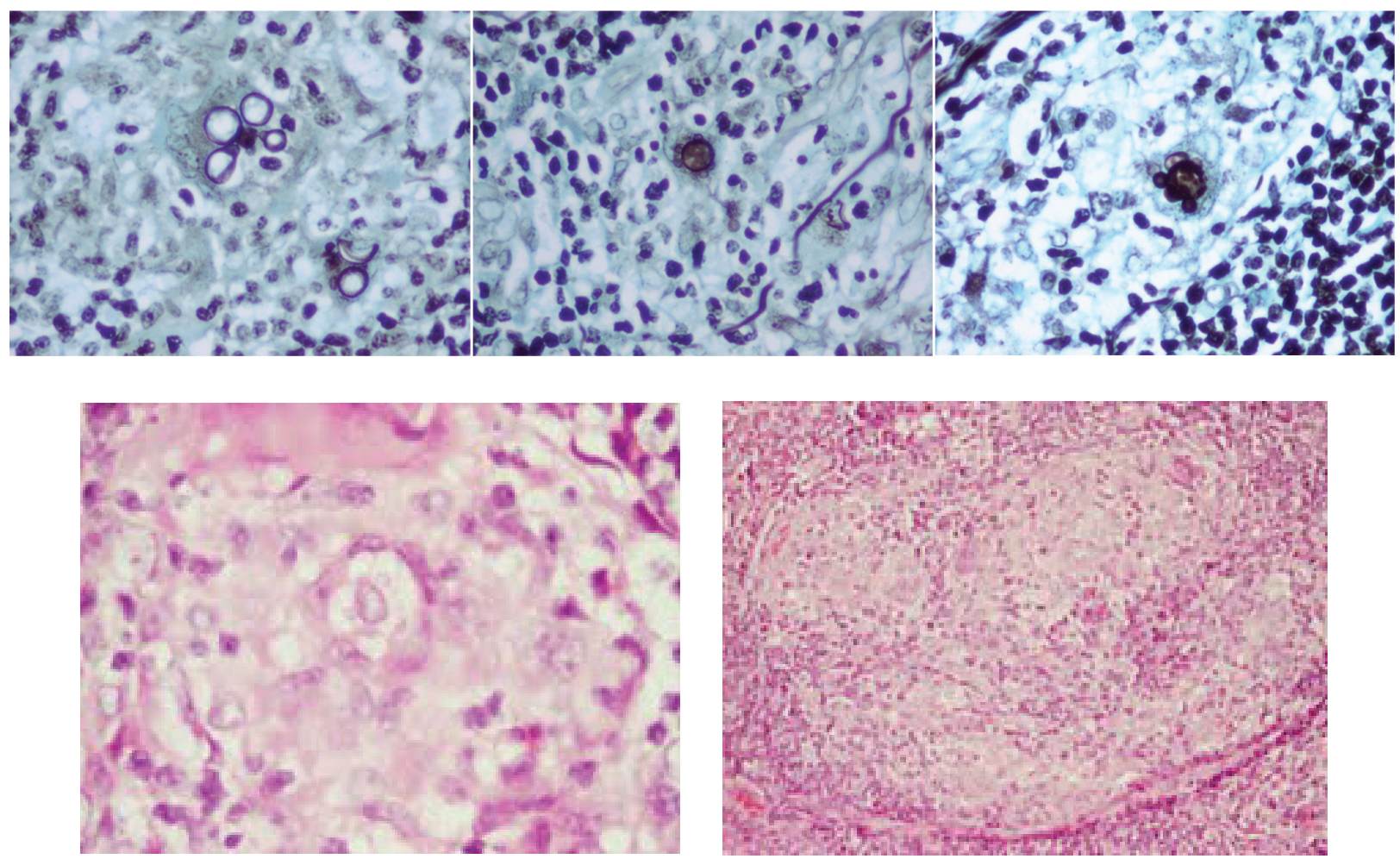

Figura 2. Biopsia de ganglio linfático. Linfadenitis granulomatosa con presencia de estructuras micóticas de paracoccidioidomicosis. Panel superior: Se observa la típica imagen de "timón de barco" de la paracoccidioidomicosis. Plata-metenamina de Grocott, 40X. Panel inferior izquierdo: A mayor aumento se identifican estructuras micóticas con gemación periférica. Hematoxilina y eosina, 40X. Panel inferior derecho: Se observan los granulomas con frecuentes eosinófilos. Hematoxilina y eosina, 40X. Cortesía del Laboratorio de Patología de la Clínica Universitaria Colombia. 
Se encontraron 95 referencias en las diferentes bases de datos: Medline (88 estudios), LILACS (cinco estudios), SciELO (dos estudios). Teniendo en cuenta la relevancia y el tipo de estudio, se seleccionaron 23 estudios por título y resumen, clasificando la información consignada para la revisión en diagnóstico, tratamiento y pronóstico.

\section{Etiología y epidemiología}

Paracoccidioides brasiliensis es un hongo con dimorfismo térmico que a $37{ }^{\circ} \mathrm{C}$ crece en forma de levadura y por debajo de $25^{\circ} \mathrm{C}$ crece en forma de micelios; en condiciones naturales, su huésped principal es el hombre, si bien la entidad ha sido informada en varias especies animales. El hábitat del hongo es desconocido, pero se piensa que la fuente de infección es la tierra contaminada con el organismo en zonas endémicas. Predomina en los habitantes de zonas rulares dedicadas a trabajos de agricultura (4). Después de la inhalación de las formas infectantes en su fase de micelio, éstas se alojan en el pulmón donde adquieren su forma levaduriforme. Este paso se considera fundamental para el establecimiento de la infección y es la fase inicial de la interacción huésped-parásito (5).

La paracoccidioidomicosis se inicia como una infección pulmonar primaria que, por lo general, pasa inadvertida y puede diseminarse por vía sanguínea o linfática a otras partes del cuerpo. La transición del estado de infección al desarrollo de la enfermedad depende del estado inmunológico del paciente, del tamaño del inóculo infeccioso y de la virulencia del hongo mismo. La inmunidad mediada por células es la principal defensa contra $P$. brasiliensis (5). En las mujeres, la infección se presenta antes de la menarquia o después de la menopausia, esto debido a que $P$. brasiliensis tiene receptores para el 17-b-estradiol en el citoplasma, y esta hormona femenina inhibe la transformación del hongo de micelio a levadura, paso indispensable para que se establezca la infección.

La forma de levadura identifica al hongo, ya que su aspecto es característico; se trata de una célula madre que se reproduce por gemación múltiple, dando lugar a varias blastoconidias y adquiriendo el aspecto típico de un timón de barco, característica de la paracoccidioidomicosis; el promedio de crecimiento de la levadura es de una semana, aproximadamente, y tanto las levaduras como los micelios son multinucleados $(4,5)$.

Las estadísticas en Colombia no se apartan de la epidemiologia de la enfermedad a nivel mundial.
En el país, la enfermedad tiene un acentuado predominio por el sexo masculino: hasta 45 casos en hombres por cada dos en mujeres (6). La prevalencia está alrededor de 2,6 por 100.000 habitantes (3), siendo un poco mayor en departamentos como Valle y Antioquia, donde se alcanzan prevalencias de 4,6 a 4,8 por 100.000 habitantes.

La descripción de casos en Colombia ha sido en adultos, al igual que la distribución general, pues no es frecuente la presentación en edad pediátrica (7).

\section{Patogénesis}

El control inmunológico de esta micosis depende de la respuesta celular inmune, la que ante la exposición al principal antígeno de $P$. brasiliensis, una glucoproteína de $43 \mathrm{kDa}$ (gp43), produce niveles de interleucinas tales como las IL2 e IL12, así como de interferón gamma los que, a su vez, activan los macrófagos y linfocitos $B$ que conforman los granulomas compactos en la respuesta inflamatoria final, estado que permite frenar la replicación rápida del hongo (8). Los pacientes que desarrollan esta enfermedad tienen déficit en la respuesta inmunológica de las células Th1 y una respuesta celular predominantemente de Th2 con activación de linfocitos $B$, hipergammaglobulinemia y títulos altos de anticuerpos específicos. Tanto en la presentación aguda como en la crónica de la afección se evidencia este fenómeno producido por las lesiones cutáneas y del tejido alveolar (9).

\section{Manifestaciones clínicas}

En la mayoría de los casos, luego de la inhalación del hongo se presenta una infección asintomática que suele entrar en latencia y cuya reactivación da lugar a síntomas, siendo ésta la forma de presentación más frecuente. Por otra parte, también puede desarrollarse la forma aguda 0 subaguda (10).

La clasificación más frecuentemente utilizada para el diagnóstico de esta enfermedad se basa en la correlación de los datos clínicos y la historia natural de la enfermedad propuesta en el Coloquio Internacional sobre Paracoccidioidomicosis realizado en Medellín en 1986, la cual permanece vigente a la fecha $(7,10)$ :

- Infección (subclínica)

- Paracoccidioidomicosis, enfermedad

- Forma aguda o subaguda (tipo juvenil)

- Forma crónica (tipo adulto)

- Forma residual 


\section{Forma aguda o subaguda (tipo juvenil)}

La forma clínica aguda y subaguda es la forma menos frecuente; generalmente ocurre en niños y adolescentes y corresponde a 5 a $10 \%$ de los casos (10). Esta presentación clínica se presenta infrecuentemente en los adultos. No tiene predilección por sexo, sin embargo, se ha visto un ligero aumento en hombres adolescentes. Su presentación y curso es de evolución rápida (4 a 12 semanas) cuando se presentan síntomas clínicos debidos a la colonización de las levaduras en el tracto respiratorio.

La enfermedad se caracteriza por la presencia de adenopatías, alteraciones digestivas, hepatoesplenomegalia, y lesiones óseas y cutáneas (10). En la mayoría de los casos se presentan síntomas inespecíficos como fiebre, pérdida de peso, anorexia y deterioro del estado general del paciente, siendo infrecuentes las manifestaciones pulmonares; de hecho, la literatura las describe solo en 10 a $20 \%$ de los casos comparados con los casos en adultos, en los cuales los síntomas respiratorios se manifiestan hasta en 70 a $90 \%$ de los casos $(6,7)$.

Las lesiones mucocutáneas se presentan en 25 $\%$ de los casos, aproximadamente; consisten en lesiones ulceradas, pueden ser vegetativas, granulomatosas o similares a las del acné. La intensidad del compromiso cutáneo depende de la gravedad del compromiso sistémico (6). Las lesiones orales y de la mucosa nasal consisten en lesiones papulosas, erosivas y, en ocasiones, sangrantes.

Son frecuentes los cambios en el hemograma; entre los hallazgos más representativos se encuentra la leucocitosis con desviación a la izquierda, la eosinofilia, la anemia y la trombocitopenia. De éstas, quizá la de mayor peso en la orientación diagnóstica es la eosinofilia, la cual se evidencia en más de $50 \%$ de los pacientes. Otros hallazgos anormales son la elevación de las enzimas hepáticas, las bilirrubinas y la proteína $C$ reactiva (PCR). En esta forma de presentación se conserva la función suprarrenal $(10,11)$.

\section{Forma crónica (tipo adulto)}

Este tipo de presentación, rara en la edad pediátrica, corresponde a $90 \%$ de todos los casos reportados (12). Se presenta principalmente en adultos de 30 a 60 años de edad y predomina en hombres con una relación de 15 a 1 . Los pacientes son, por lo general, personas que trabajan en la agricultura, y las manifestaciones clínicas se presentan meses o inclusive años después de la infección primaria (13).

Cualquier órgano o sistema puede verse afectado. Su evolución es de progreso lento, silente, lo cual determina que, en la mayoría de casos, su diagnóstico tome años (14). A diferencia de lo que ocurre con la forma aguda, este tipo de presentación tiene como principal manifestación el compromiso pulmonar, el cual se encuentra hasta en $90 \%$ de los pacientes (15), en los cuales la disnea y la tos seca son síntomas predominantes que pueden llegar a comprometer de manera extensa el pulmón, incluida la fibrosis pulmonar (16). El compromiso de mucosas se calcula hasta en $50 \%$ de todas las formas de presentación, siendo la cavidad oral el sitio más afectado (17). Menos común (25\%) es la afección en piel (6). También puede existir compromiso de los ganglios linfáticos, el sistema nervioso central y las glándulas suprarrenales $(18,19)$.

Los hallazgos radiológicos característicos son la presencia de infiltrados intersticiales y alveolares de compromiso simétrico; se pueden encontrar también cavernas (20).

\section{Diagnóstico diferencial}

El diagnóstico diferencial se hace con entidades clínicas que tengan características de presentación similar, entre las que se destacan la histoplasmosis diseminada, la tuberculosis, la sífilis, la enfermedad por arañazo de gato, el síndrome similar a la mononucleosis (virus de Epstein-Barr, citomegalovirus, Toxoplasma gondii), y el VIH (21).

\section{Diagnóstico}

El diagnóstico de la paracoccidioidomicosis se basa en la identificación de $P$. Brasiliensis (21), el cual se puede encontrar en diferentes muestras clínicas como esputo, exudado de lesiones mucocutáneas, biopsia ganglionar o biopsia de las lesiones en piel. En más de $90 \%$ de los casos, la visualización directa del hongo suele lograse con el examen en fresco con hidróxido de potasio $(\mathrm{KOH})(11,22)$.

El cultivo debe realizarse en un medio adecuado, especialmente en agar de dextrosa Sabouraud - agar de extracto de levadura que contenga cloranfenicol y cicloheximida, en los cuales el hongo puede tardar de 20 a 30 días en crecer (11).

Las pruebas serológicas también pueden emplearse en el diagnóstico, pero su importancia principal radica en el seguimiento de la respuesta al 
tratamiento instaurado (22). Entre las pruebas serológicas usadas están la doble inmunodifusión en gel de agar, la inmunofluorescencia indirecta (IFI) y las pruebas ELISA y de inmunotransferencia, las cuales muestran sensibilidades de entre 85 y $100 \%$.

En las pruebas de inmunodifusión cualquier resultado positivo se considera diagnóstico de la enfermedad; éstas también tienen utilidad en el seguimiento de la respuesta al tratamiento (22).

La reacción en cadena de la polimerasa para la amplificación de ADN tiene muy buena sensibilidad y especificidad, pero todavía no hace parte de las pruebas diagnósticas usuales, dado el acceso limitado a ella (23).

\section{Tratamiento}

Paracoccidioides brasiliensis continúa siendo sensible a la gran mayoría de antifúngicos disponibles (24). La duración del tratamiento depende de la gravedad de la enfermedad y del tipo de medicamento utilizado; en la gran mayoría de casos, estos tratamientos son de larga duración, con lo que se permite el adecuado control de las manifestaciones clínicas y se evitan, además, las recaídas.

Las sulfamidas fueron los primeros fármacos utilizados para el tratamiento de las micosis, y continúan siendo efectivas; la combinación de trimetoprim y sulfametoxazol es la forma más usada; la presentación intravenosa se reserva para las presentaciones graves de la enfermedad. A finales de la década de los 70, con el advenimiento de los imidazoles, el manejo de esta entidad cambió, pues se demostró que su administración oral tenía excelente actividad contra $P$. brasiliensis, era bien tolerado y tenía pocos efectos secundarios.

Las guías para el tratamiento de la paracoccidioidomicosis recomiendan el tratamiento basado en el criterio de la gravedad (10), como se describe a continuación.

- Enfermedad leve a moderada: manejo con itraconazoloral, $100 \mathrm{mg}$, una o dos veces al día en adultos; en niños, solución oral de $5 \mathrm{mg} / \mathrm{kg}$ una vez al día, o trimetoprim-sulfametoxazol, $10 \mathrm{mg} / \mathrm{kg}$ al día.

- Enfermedad grave: manejo con anfotericina B, 0,7 a $1 \mathrm{mg} / \mathrm{kg}$ al día, o trimetoprim-sulfametoxazol intravenoso, 8 a $10 \mathrm{mg} / \mathrm{kg}$ al día.

Una vez resueltos los signos de gravedad (resolución de la hipotensión, ascitis, mejoría de la función respiratoria y del estado nutricional), lo que habitualmente se consigue después de 20 a 40 días de terapia intravenosa, se puede cambiar al esquema oral.

La duración de la terapia depende del agente farmacológico elegido y de la gravedad de la enfermedad. Si se emplea trimetoprim-sulfametoxazol, la duración generalmente será mayor a dos años. $\mathrm{Si}$ se eligen azoles (itraconazol, voriconazol), la duración puede ser de seis a 12 meses. No obstante, si se trata de una forma grave, el tratamiento debe prolongarse durante al menos dos años, así el agente elegido sean los azoles. Se recomienda continuar el tratamiento hasta la resolución clínica y radiológica completa.

Se deben realizar pruebas serológicas para monitorizar la respuesta al tratamiento antes del inicio de la terapia, a los tres meses y cada seis meses hasta la finalización del tratamiento.

Las recaídas se producen en menos del $5 \%$ de los pacientes tratados con itraconazol y, generalmente, se relacionan con presentaciones crónicas de la enfermedad y en aquellos pacientes que no recibieron el tratamiento completo. La tasa de recaída aumenta a $25 \%$ en los pacientes tratados con trimetoprim-sulfametoxazol (11).

\section{Criterios de curación}

SI bien la paracoccidioidomicosis no es totalmente curable, ya que el hongo permanece latente pero viable por décadas, incluso después de un tratamiento adecuado, los criterios de mejoría estable se basan en características clínicas, radiológicas e inmunológicas (25).

En el espectro clínico se encuentran la resolución de las lesiones en piel, la regresión de las linfadenopatías, la recuperación del peso y la desaparición de los síntomas generales. Asimismo, se considera criterio de recuperación la desaparición o mejoría notable de las lesiones radiológicas, con estabilización de secuelas cicatriciales y hallazgos de tipo inmunológico como son la negativización de los títulos en la prueba de doble inmunodifusión y la estabilización a niveles bajos de los títulos de anticuerpos medidos por la fijación del complemento o por ELISA (13).

\section{Pronóstico}

El pronóstico de la enfermedad depende de la gravedad y del agente terapéutico empleado, así como de las enfermedades concomitantes existentes. La presentación aguda tiene mayores 
tasas de mortalidad que la crónica; sin embargo, la presentación crónica presenta más secuelas a largo plazo $(13,25)$.

\section{Discusión}

Se presenta el reporte del caso de una niña de 12 años inmunocompetente con paracoccidioidomicosis aguda diseminada, procedente de zona urbana, que consultó por presencia de un síndrome febril, compromiso ganglionar generalizado y manifestaciones cutáneas.

Si bien la paracoccidioidomicosis es una de las afecciones micóticas sistémicas más prevalente en países latinoamericanos, son pocos los casos de paracoccidioidomicosis en la población pediátrica, mucho menos aun que los reportados en zonas urbanas en poblaciones sin factores de riesgo. Los cambios demográficos han llevado a la "urbanización" de $P$. brasiliensis, inclusive a la presentación de esta micosis en pacientes en los cuales no se encuentran asociaciones causales.

La detección directa de $P$. brasiliensis es una herramienta diagnóstica clave. Actualmente, las pruebas serológicas se usan en el diagnóstico, pero tienen mayor utilidad en el seguimiento de la mejoría que sigue al tratamiento. En nuestra paciente el diagnóstico se realizó mediante la visualización de las estructuras fúngicas típicas en la biopsia de ganglio confirmada por técnicas moleculares como la amplificación y secuenciación del hongo, que fue comparado con los bancos de datos $y$, finalmente, identificado plenamente como $P$. brasiliensis (Corporación para Investigaciones Biológicas, Medellín). La paciente presentó mejoría clínica y paraclínica. Completó 16 meses de tratamiento, durante los cuales no se han observado efectos secundarios ni sospecha de recaída.

En la población pediátrica, la sintomatología es bizarra y puede simular otras etiologías; sin embargo, el abordaje minucioso e integral del síndrome febril debe contemplar esta causa, dado que debido a múltiples factores, entre ellos los cambios climáticos, la epidemiología de las enfermedades infecciosas tropicales ha cambiado, por lo que es necesario que los médicos no olviden esta enfermedad usual en los trópicos de América Latina.

\section{Agradecimientos}

Al Departamento de Pediatría de la Clínica Universitaria Colombia por permitir la publicación de este estudio; al Departamento de Patología de la Clínica Universitaria Colombia por la información suministrada en el desarrollo de este estudio; a la doctora Ángela Restrepo Moreno por la revisión del artículo y su invaluable asesoría para el diagnóstico de las enfermedades micóticas en nuestro país, y al doctor Carlos E. Pinzón por su asesoría en el desarrollo de la revisión de la literatura.

\section{Conflicto de intereses}

Los autores declaran no presentar ningún conflicto de intereses.

\section{Financiación}

Este estudio no tuvo financiación por parte de organismos o instituciones públicas o privadas.

\section{Referencias}

1. Ameen $\mathbf{M}$, Talhari $\mathbf{C}$, Talhari $\mathbf{S}$. Advances in paracoccidioidomycosis. Clin Exp Dermatol. 2010;6:57680. http://dx.doi.org/10.1111/j.1365-2230.2009.03647.x

2. Burstein-Alva Z. Aspectos clínicos de la blastomicosis sudamericana (Paracoccidioidomicosis) en el Perú. Rev Peru Med Exp Salud Publica. 2002;19:43-7.

3. Ferreira MS. Paracoccidioidomycosis. Paediatr Respir Rev. 2009;4:161-5. http://dx.doi.org/10.1016/j.prrv.2009.08.001

4. Blotta MH, Mamoni RL, Oliveira SJ, Nouér SA, Papaiordanou PM, Goveia A, et al. Endemic regions of paracoccidioidomycosis in Brazil: A clinical and epidemiologic study of 584 cases in the Southeast region. Am J Trop Med Hyg. 1999;61:390-4.

5. Brummer E, Castañeda E, Restrepo A. Paracoccidioidomycosis: An update. Clin Microbiol Rev. 1993;6:89-117. http://dx.doi.org/10.1128/CMR.6.2.89

6. Giraldo R, Restrepo A, Gutiérrez F, Robledo M, Londoño $\mathrm{F}$, Hernández $\mathrm{H}$, et al. Pathogenesis of paracoccidioidomycosis. A model based in the study of 46 patients. Mycopathologia. 1976;58:63-70.

7. Greer LD, Restrepo A. La epidemiología de la paracoccidioidomicosis. Bol Of San Panam. 1977;82:428-45.

8. Marques Mello L, Silva-Vergara ML, Rodrigues V Jr. Patients with active infection with Paracoccidioides brasiliensis present a Th2 immune response characterized by high Interleukin-4 and Interleukin-5 production. Hum Immunol. 2002;63:149-54. http://dx.doi.org/10.1016/S01988859(01)00375-5

9. Mamoni RL, Blotta MH. Kinetics of cytokines and chemokines gene expression distinguishes Paracoccidioides brasiliensis infection from disease. Cytokine 2005;32:20-9. http://dx.doi.org/10.1016/j.cyto.2005.07.006

10. Shikanai-Yasuda MA, Telles Filho F, Pôncio Mendes R, Lopes Colombo A, Moretti M, Grupo de Consultores do Consenso em Paracoccidioidomicose. Consenso em paracoccidioidomicose. Rev Soc Bras Med Trop. 2006;39: 297-310. http://dx.doi.org/10.1590/S0037-86822006000300017

11. Restrepo A, Tobón AM, Agudelo CA. Paracoccidioidomycosis. En: Hospenthal DR, Rinaldi MG, editors. Diagnosis and treatment of human mycoses. 1st edition. Totowa, NJ: Humana Press; 2008. 
12. Travassos LR, Taborda CP, Colombo AL. Treatment options for paracoccidioidomycosis and new strategies investigated. Expert Rev Anti Infect Ther. 2008;6:251-62. http://dx.doi.org/10.1586/14787210.6.2.251

13. Pereira RM, Bucaretchi F, Barison Ede M, Hessel G, Tresoldi AT. Paracoccidioidomycosis in children: Clinical presentation, follow-up and outcome. Rev Inst Med Trop Sao Paulo. 2004;46:127-31. http://dx.doi.org/10.1590/S003646652004000300002

14. Hospenthal DR, Rinaldi MG. Diagnosis and treatment of human mycoses. 1st edition. Totowa, NJ: Humana Press; 2008.p.127-331.

15. Restrepo A, Benard G, de Castro CC, Agudelo CA, Tobón AM. Pulmonary paracoccidioidomycosis. Semin Respir Crit Care Med. 2008;29:182-97. http://dx.doi.org/10.1055/s-20081063857

16. Correa AL, Franco L, Restrepo A. Paracoccidioidomicosis: coexistencia de lesiones pulmonares y patología pulmonar silente. Acta Med Colomb. 1991;16:304-8.

17. Bicalho RN, Santo MF, de Aguiar MC, Santos VR. Oral paracoccidioidomycosis: A retrospective study of 62 Brazilian patients. Oral Dis. 2001;7:56-60. http://dx.doi. org/10.1034/j.1601-0825.2001.70111.x

18. Paniago AM, de Oliveira PA, Aguiar ES, Aguiar JI, da Cunha RV, Leme LM, et al. Neuroparacoccidioidomycosis: Analysis of 13 cases observed in an endemic area in Brazil. Trans R Soc Trop Med Hyg. 2007;101:414-20. http://dx.doi. org/10.1016/j.trstmh.2006.07.006
19. Colombo AL, Faiçal S, Kater CE. Systematic evaluation of the adrenocortical function in patients with paracoccidioidomycosis. Mycopathologia. 1994;127:89-93.

20. Tobón AM, Agudelo CA, Osorio ML, Álvarez DL, Arango M, Cano LE, et al. Residual pulmonary abnormalities in adult patients with chronic paracoccidioidomycosis: Prolonged follow-up after itraconazole therapy. Clin Infect Dis. 2003;37:898-904. http://dx.doi.org/10.1086/377538

21. Nucci M,Colombo AL. Clinical manifestations and diagnosis of acute/subacute paracoccidioidomycosis. UpToDate. 2012. Fecha de consulta: 15 de octubre de 2012. Disponible en: http://www.uptodate.com/contents/clinical-manifestationsand-diagnosis-of-acute-subacute-paracoccidioidomycosis.

22. De Camargo ZP. Serology of paracoccidioidomycosis. Mycopathologia. 2008;165:289-302.

23. Motoyama AB, Venancio EJ, Brandão GO, PetrofezaSilva S, Pereira IS, Soares CM, et al. Molecular identification of Paracoccidioides brasiliensis by PCR amplification of ribosomal DNA. J Clin Microbiol. 2000;38:3106-9.

24. Gomes GM, Cisalpino PS, Taborda CP, de Camargo ZP. PCR for diagnosis of paracoccidioidomycosis. J Clin Microbiol. 2000;38:3478-80.

25. Menezes VM, Soares BG, Fontes CJ. Drugs for treating paracoccidioidomycosis. Cochrane Database Syst Rev. 2006;CD004967. http://dx.doi.org/10.1002/14651858. CD004967.pub2 